Caso clínico

\title{
ABORDAJE HOLÍSTICO DE LAS LESIONES POR PRESIÓN EN LA PERSONA MAYOR: CASO CLÍNICO
}

\author{
HOLISTIC APPROACH TO PRESSURE INJURIES IN THE ELDERLY: CLINICAL \\ CASE
}

\author{
Miguel Andrez Valencia Contrera \\ Enfermero \\ Licenciado en Enfermería, Magíster (c) en Enfermería, Universidad de Antofagasta, \\ Departamento de Enfermería, Antofagasta, Chile \\ m.valencia@outlook.cl \\ https://orcid.org/0000-0002-4943-5924
}

Artículo recibido el 11 de octubre de 2021. Aceptado en versión corregida el 26 de noviembre de 2021.

\section{RESUMEN}

Se expone un caso clínico de una paciente de 88 años cursando una hospitalización domiciliaria de larga estadía, por múltiples lesiones por presión, tras su seguimiento presenta una merma en su recuperación, por lo que un profesional de enfermería de hospitalización domiciliaria realiza una visita domiciliaria integral que evidencia múltiples elementos que precisan un abordaje holístico de la situación. Para su abordaje se utilizó el marco teórico de Virginia Henderson, que facilitó la elección del diagnóstico en la cuidadora de Cansancio del rol de cuidador, lo cual permitió que la situación lograra ser abordada satisfactoriamente. CONCLUSIÓN: se relevó la importancia de un manejo holístico en las lesiones por presión, que debe considerar la dimensión social en la que está inmerso el paciente, junto a un manejo interdisciplinario, preparación profesional y abordaje precoz del probable impacto económico en estos pacientes.

Palabras clave: Informe de casos, manejo de caso, úlcera por presión, enfermería geriátrica, atención de enfermería, proceso de enfermería, Enfermería Holística (DeCS).

\begin{abstract}
A clinical case of an 88-year-old patient undergoing a long-term home hospitalization due to multiple pressure injuries is presented. After follow-up, she presents a decline in her recovery, so a home hospitalization nursing professional performs a comprehensive home visit that shows multiple elements that require a holistic approach to the situation. For its approach, the theoretical framework of Virginia Henderson was used, which facilitated the choice of the diagnosis in the caregiver of Tiredness from the caregiver role, which allowed the situation to be satisfactorily addressed. CONCLUSION: the importance of a holistic management of pressure injuries was highlighted, which must consider the social dimension
\end{abstract}


Abordaje holístico de las lesiones por presión en la persona mayor: Caso clínico in which the patient is immersed, together with an interdisciplinary management, professional preparation, and an early approach to the probable economic impact on these patients.

Keywords: Case reports, case management, pressure ulcer, geriatric nursing, nursing care, nursing process, Holistic Nursing (DeCS).

\section{http://dx.doi.org/10.7764/Horiz_Enferm.32.3.341-351}

\section{INTRODUCCIÓN}

La Lesión por Presión (LPP) es definida como "el daño localizado en la piel y/o en el tejido blando subyacente, habitualmente sobre una prominencia ósea o derivado de un dispositivo médico o de otro tipo. $\mathrm{La}$ piel de la lesión puede presentarse como piel intacta o como úlcera abierta y ser dolorosa. Se produce como resultado de una presión intensa y/o prolongada o de la conjunción de presión y fuerzas de cizalla. En la tolerancia de los tejidos blandos a la presión y la cizalla también pueden influir el microclima, la nutrición, la perfusión, las comorbilidades y el estado de dichos tejidos blandos"(1).

Antiguamente las LPP se denominaban "Úlceras por Presión" (UPP), sin embargo, se consideró que el término "úlcera" no describía con exactitud la LPP de la categoría 1, vale decir, aquel estadio donde aún no existe perdida de la continuidad de la integridad cutánea, ya que una úlcera en estricto rigor no puede estar presente sin una lesión. No obstante, una lesión puede estar presente sin una úlcera. Dicho cambio de nomenclatura ocurre en el año 2016, durante la Conferencia de Consenso sobre la categorización de la National Pressure Ulcer Advisory Panel (NPUAP), llevada a cabo en Rosemont, Illinois, el cual está incluido en las guías de práctica clínica basadas en evidencia de "Registred Nurse Association of Ontario" (RNAO) ${ }^{(2)}$, donde Chile tiene convenio de acuerdo para la implementación de estas en su territorio desde el 11 de diciembre de $2017^{(3)}$.

Sin perjuicio de lo señalado, es pertinente señalar que se ha descrito por parte de la comunidad científica el uso mantenido de su nomenclatura antigua ${ }^{(4)}$, cuya etiología probable puede ser atribuida a la resistencia mantenida del uso de "Úlcera por Presión" por parte de la Clasificación Internacional de Enfermedades (CIE), cuya última versión, la número 11, entró en vigor a partir de enero del año 2021(5); sin embargo, dicha clasificación manifiesta la inclusión del término LPP, aun cuando el diagnóstico sigue siendo "Úlcera por Presión".

En cuanto a la epidemiología de la enfermedad, se ha descrito a nivel intrahospitalario una incidencia del $7 \%$, a nivel domiciliario hasta un $17 \%$ y en establecimientos de larga estancia hasta un $25 \%{ }^{(6)}$. En lo referente a las localizaciones más frecuentes de las LPP, se ha descrito la región sacra, glúteos, talones y trocánter, de mayor a menor frecuencia respectivamente ${ }^{(7)}$. Las principales medidas para prevenir la patología son variadas y copiosas (Tabla 1), estas deben adaptarse a la realidad de cada paciente, siempre 
considerando la integralidad, un enfoque holístico y, además, deben involucrar tanto a los pacientes con factores riesgo (Tabla 2) como a sus familias ${ }^{(8)}$.

Tabla 1. Principales medidas de prevención de las Lesiones por Presión (LPP). Inspección diaria de la piel.

Aseo con productos neutros, para minimizar la irritación y resequedad de la piel.

Aplicar agentes hidratantes.

Evitar el masaje sobre las prominencias óseas.

Evitar exposición de la piel a la orina, materia fecal, transpiración y drenaje de la herida.

Reducir al mínimo la fricción y rozamiento: postura, transferencia y cambios de posición, idealmente cada 2 a 3 hrs.

Prevenir la malnutrición, y estimular la movilización.

En pacientes postrados, realizar ejercicios pasivos y activos, y posturas que disminuyan la presión sobre prominencias óseas.

Uso de colchón anti escaras, de protectores que disminuyan la presión.

Fuente: Ministerio de Salud. Actualización manual de geriatría para médicos. $1^{\mathrm{a}}$

ed. Santiago, Chile: MINSAL; 2019. ISBN: 978-956-348-182-2

Tabla 2. Factores de riesgo de las Lesiones por Presión (LPP).

Edad avanzada.

Enfermedades crónicas: insuficiencia cardiaca, EPOC, diabetes mellitus, insuficiencia

hepática y renal, etc.

Desnutrición, bajo peso.

Demencia avanzada.

Incontinencia urinaria o fecal.

Inmovilismo, hospitalizaciones prolongadas, requerimiento de sondaje permanente.

Mala higiene, estrés del cuidador.

Fuente: Ministerio de Salud. Actualización manual de geriatría para médicos. $1^{\text {a }}$

ed. Santiago, Chile: MINSAL; 2019. ISBN: 978-956-348-182-2

\section{CASO CLÍNICO}

Paciente femenina de 88 años, con antecedentes mórbidos de Diabetes Mellitus tipo 2 y demencia senil, actualmente se encuentra postrada y dependiente completamente de las Actividades Básicas de la Vida Diaria (ABVD). Presenta una hospitalización domiciliaria de larga estadía por múltiples LPP y vive con su familia, compuesta por una de sus hijas y con algunos de sus nietos. La hija es la cui- dadora responsable, quien manifiesta que "ninguno de los demás hermanos se preocupa por su mamá", por lo que se siente un tanto agobiada. En la revisión de su historial clínico, se evidencia un plan de cuidado diario y otro cada tres días (debido a los diferentes tipos de lesiones que presenta). Se destaca dentro de los registros la inasistencia de su cuidador responsable en reiteradas ocasiones, 
Abordaje holístico de las lesiones por presión en la persona mayor: Caso clínico

motivo por el cual, no se ha cumplido el plan de cuidados según lo programado $\mathrm{y}$, por consiguiente, los criterios de resultados están medianamente cumplidos y otros incumplidos.

Se realiza una Visita Domiciliaria Integral (VDI) para obtener mayores antecedentes del caso la que destaca buenas condiciones higiénicas de la vivienda $\mathrm{y}$, habitación de la paciente con cama clínica, sin embargo, el colchón anti escara está en mal estado. Al respecto, la cuidadora refiere estar esperando por un tiempo prolongado su cambio, ya que una organización de apoyo social se comprometió en proporcionárselo; velador con restos de postres envasados, pues según lo referido son sus favoritos, mal olor en la habitación, escasa ventilación y una merma en la luminosidad de la misma.

Al examen físico segmentado céfalo-caudal se destaca: posición y decúbito, paciente en decúbito dorsal indiferente, una facie no característica, desorientada temporoespacialmente, constitución física ectomórfica con una sarcopenia importante, turgor y elasticidad concordante con la edad. Al control de signos vitales: normocardica con pulsos cuya amplitud era normal, ritmo regular, con simetría entre extremidades contralaterales y entre extremidades superiores e inferiores; normotensa, eupneica, respiración costal, con ritmo regular y amplitud disminuida, normosaturando y afebril.

Normocráneo, pelo de distribución ginecoide y graso, se destaca resequedad de la piel, globo ocular con arco senil, se evidencia falta de piezas dentales, abundante tártaro interdental y halitosis. Cuello con piel indemne, movimiento sin alteraciones y yugulares no ingurgitadas.
Tórax con una configuración normal, expansión y elasticidad conservada, se destaca lesión en regresión ubicada en clavícula izquierda (Figura 1).

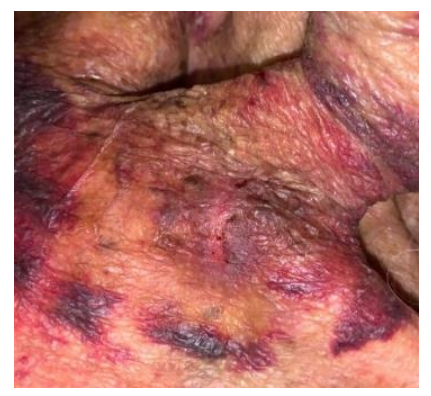

Figura 1. Clavícula izquierda.

Lesión cien por ciento tejido granulatorio, extensión de $2 \mathrm{~cm}$ de largo y $0,5 \mathrm{~cm}$ de ancho aproximadamente, piel circundante frágil, dolor en la zona. Se realiza curación avanzada, quedando fijo, limpio y seco; columna con escoliosis. Examen pulmonar con vibraciones vocales positivas y simétricas, sonoridad normal a la percusión, simétrico en ambos campos, murmullo vesicular positivo, sin ruidos adventicios.

Abdomen de configuración normal, a la inspección se observa color de piel sin diferencias en la pigmentación, distribución uniforme de vello, ruidos hidroaéreos positivos, abdomen blando, depresible, no se palpan masas.

Miembros superiores, se evidencia simetría en ambos miembros, ausencia de deformidades. Sin embargo, se identifican dos lesiones ubicadas en el tercio medio de antebrazo izquierdo (Figura 2), lesión con escaso exudado, turbio sin mal olor, con tejido cien por ciento granulatorio, extensión de $2 \mathrm{~cm}$ de largo y $1 \mathrm{~cm}$ de ancho, y $1 \mathrm{~cm}$ de largo y $1 \mathrm{~cm}$ de ancho aproximadamente con piel circundante reseca, zona dolorosa. Se realiza curación 
avanzada, dejando fijo, limpio y seco. Uñas largas y sucias.

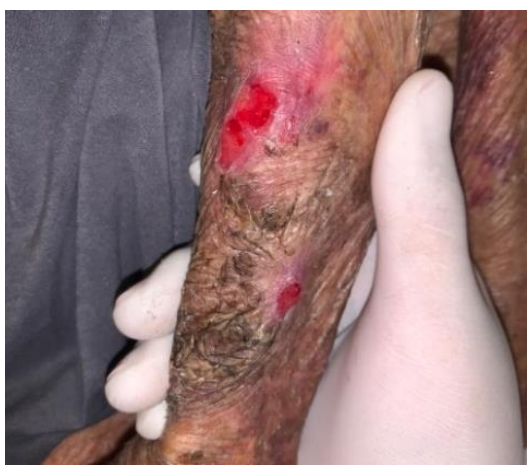

Figura 2. Tercio medio de antebrazo izquierdo.

Miembros inferiores evidencian simetría, coloración de la piel uniforme, ausencia de

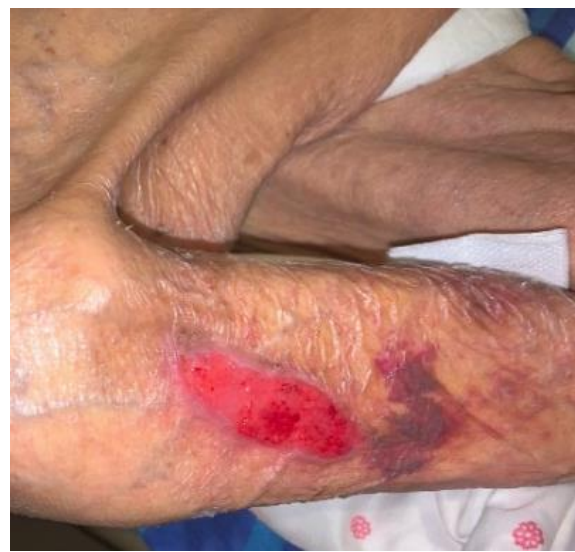

Figura 4. Zona lateral del tercio proximal de la región tibial izquierda.

LPP en región trocantérica izquierda: a la valoración escaso exudado, turbio sin mal olor, lesión con tejido hipergranulado, extensión aproximada de $15 \mathrm{~cm}$ de largo y $10 \mathrm{~cm}$ de ancho, piel circundante con zonas maceradas, ausencia de dolor. Se realiza curación avanzada, dejando fijo, limpio y seco. LPP en región tibial izquierda, tercio proximal: escaso exudado hemático, con tejido granulatorio, sangrante, herida en regresión, cuya extensión es de $6 \mathrm{~cm}$ de largo y $2 \mathrm{~cm}$ de ancho deformidades. No obstante, se identifican tres lesiones: en la región trocantérica izquierda (Figura 3), zona lateral del tercio proximal de la región tibial izquierda (Figura 4) y talón izquierdo (Figura 5).



Figura 3. Región trocantérica izquierda.

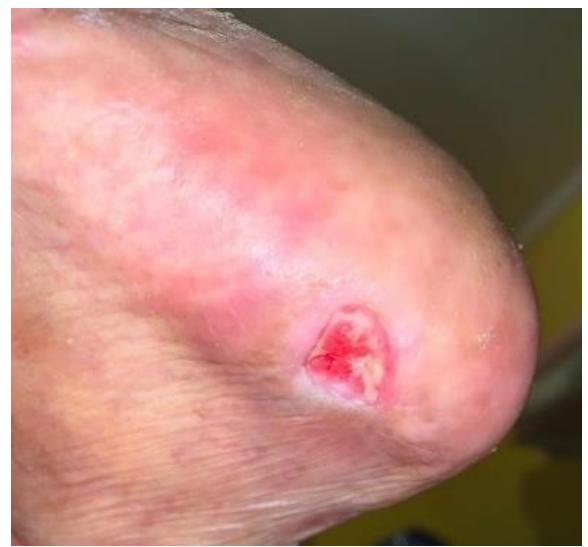

Figura 5. Talón izquierdo.

aproximadamente, piel circundante indemne, dolor en la zona, se realiza curación avanzada, quedando fijo, limpio y seco. LPP en talón izquierdo: escaso exudado, turbio sin mal olor, con tejido granulatorio, escaso tejido esface-lado, extensión de $2 \mathrm{~cm}$ de largo por $1 \mathrm{~cm}$ de ancho aproximadamente, piel circun-dante indemne, dolor al tacto. Se realiza curación avanzada, dejando fijo, limpio y seco. 
Abordaje holístico de las lesiones por presión en la persona mayor: Caso clínico

Zona sacra con LPP (Figura 6), lesión circular con escaso exudado turbio sin mal olor, zonas con tejido hipergranulado, extensión de $10 \mathrm{~cm}$ de diámetro aproximadamente, piel circundante indemne, ausencia de dolor. Se realiza curación avanzada quedando fijo, limpio y seco.

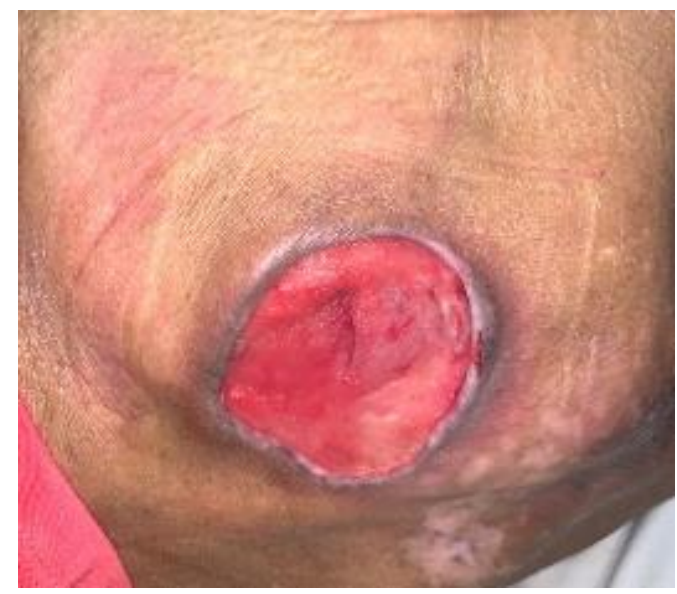

Figura 6. Zona sacra.

PRIORIZACIÓN DE PROBLEMAS

Para el abordaje de la situación en salud, se utilizó el marco teórico de Virginia Henderson $^{(9)}$, pues existen estudios que respaldan su aporte en la evaluación de los cuidados domiciliarios de enfermería a pacientes ancianos ${ }^{(10)}$. A continuación, se describe el problema en salud prioritario y su fundamento:

Es correcto pensar que el problema prioritario son las lesiones de la paciente en cuestión. Debido a ello, es pertinente dejar en manifiesto que dicho problema fue abordado en un Proceso de Enfermería ajustado a un nuevo plan; sin embargo, es necesario introducirnos en el plano multidimensional en el que está inmersa la paciente, puesto que la problemática no está circunscrita a las múltiples lesiones con perdida de la integridad cutánea. Más bien, lo que debe llamar la atención a cualquier profesional de enfermería, es la discontinuidad del Proceso de Enfermería que ya estaba en curso con la paciente, lo que nos hace cambiar el foco de atención, centrándonos en su cuidadora.

En la valoración descrita, se evidencian pequeños rasgos de la cuidadora, pero que comunican un poderoso mensaje, tales como:

- Existen registros que dan cuenta de la inasistencia de la cuidadora en el hogar, por consiguiente, la incapacidad de mantener el plan de cuidados establecido.

- La cuidadora refiere presentar más hermanos, los cuales no son partícipes del cuidado de la paciente, sobrecargando su rol.

- Barrera económica, al tener que acudir a solicitar apoyo social, sin recibirlo.

- Se evidencia desconocimiento, al proporcionar alimentos con elevado contenido glucémico, en contexto de una paciente con Diabetes Mellitus tipo 2. 
PROCESO DE ENFERMERÍA

Diagnóstico de enfermería prioritario Cansancio del rol de cuidador [00061] $]^{(11)}$; relacionado con, duración extendida de la prestación de cuidado requerido, asistencia inadecuada, ambiente físico inadecuado para brindar atención, patrón de disfuncionalidad familiar, apoyo social inadecuado, proveedor de cuidados de sexo femenino, enfermedad crónica y disfunción cognitiva en el receptor de cuidado; manifestado por, dificultad por completar las tareas requeridas, dificultad para llevar a cabo las tareas requeridas, tiempo insuficiente para satisfacer las necesidades personales y conflicto familiar.

Objetivo del proceso de enfermería Preparación del cuidador familiar domiciliario [2202] ${ }^{(12)}$. El cuidador principal demostrará presentar preparación para cumplir su rol en el transcurso de tres semanas. Indicadores ${ }^{(12)}$ :

- [220202] Conocimiento sobre el rol de cuidador.

- [220206] Conocimiento del régimen de tratamiento recomendado.

- [220205] Conocimiento del proceso de enfermedad del receptor de los cuidados.

- [220212] Idoneidad de los recursos económicos.

- [220214] Apoyo social.

- [220213] Conocimiento de las visitas a los profesionales sanitarios.

Intervenciones: Solo se expondrán las principales actividades por intervención debido al menguado espacio disponible para ello, siguiendo las orientaciones de la Clasificación de Intervenciones en Enfermería (NIC) ${ }^{(13)}$ :

\section{Apoyo al cuidador principal [7040]}

1. Aceptar las expresiones de emoción negativa.

2. Estudiar junto con el cuidador los puntos fuertes y débiles.

3. Animar la aceptación de interdependencia entre los miembros de la familia.

4. Enseñar técnicas de cuidado para mejorar la seguridad del paciente.

5. Animar al cuidador a participar en grupos de apoyo.

\section{Aumentar los sistemas de apoyo [5440]}

1. Fomentar las relaciones con personas que tengan los mismos intereses $\mathrm{y}$ metas.

2. Determinar los puntos fuertes y débiles de los recursos comunitarios y aconsejar los cambios cuando sean apropiados.

3. Remitir a programas comunitarios de prevención o tratamiento, según corresponda.

4. Implicar a la familia/allegados/amigos en los cuidados y la planificación.

5. Explicar a los demás implicados la manera en que pueden ayudar.

Manejo ambiental: preparación del hogar [6485]

1. Encargar y validar el funcionamiento de cualquier equipo necesario.

2. Encargar y confirmar la entrega de cualquier medicación y suministros necesarios.

3. Proporcionar información escrita respecto a la medicación, los suministros y los dispositivos de ayuda como guía para la familia, según sea necesario. 
Abordaje holístico de las lesiones por presión en la persona mayor: Caso clínico

4. Organizar el programa del personal de apoyo.

5. Realizar un seguimiento para asegurarse de que los programas son factibles y se llevaron a cabo.

\section{Orientación en el sistema sanitario [7400]}

1. Ayudar a la familia a coordinar la asistencia sanitaria y la comunicación.

2. Informar al paciente sobre los recursos comunitarios adecuados y las personas de contacto.

3. Determinar y facilitar la comunicación entre los profesionales sanitarios y la familia.

4. Ayudar a los pacientes a rellenar los formularios de asistencia, tales como de ayuda económica, si lo precisan.
5. Notificar a la familia de las visitas programadas, según corresponda.

Resultados esperados: El cuidador principal demuestra presentar preparación para cumplir su rol al cabo de tres semanas. Evidenciado a través del cumplimiento de los indicadores propuestos (Conocimiento sobre el rol de cuidador, Conocimiento del régimen de tratamiento recomendado, Conocimiento del proceso de enfermedad del receptor de los cuidados, Idoneidad de los recursos económicos, y Apoyo social), a través de la aplicación de la escala "grado de suficiencia en cantidad o calidad para conseguir un estado deseado"(12), cuyo puntaje mínimo requerido para considerar el cumplimiento de cada indicador será de 3 , en una escala de 1 a 5 (Tabla 3 ).

Tabla 3. Grado de suficiencia en cantidad o calidad para conseguir un estado deseado.

\begin{tabular}{l}
\hline 1. Inadecuado \\
\hline 2. Ligeramente adecuado \\
\hline 3. Moderadamente \\
\hline 4. Sustancialmente adecuado \\
\hline 5. Completamente adecuado \\
\hline Fuente: Moorhead S, Swanson E, Johnson M, Maas M, \\
Echevarría Pérez P, Morales Moreno I, et \\
al. Clasificación de resultados de enfermería (NOC). $6^{\text {a }}$ \\
ed. Barcelona: Elsevier España; 2018.
\end{tabular}

\section{DISCUSIÓN}

Dada la complejidad y la heterogeneidad de los factores involucrados en el proceso salud-enfermedad, es que cada vez amerita más su abordaje desde una esfera holística, una visión que enfermería ha demostrado tener desde el inicio de su existencia, proporcionando un paradigma disímil a cualquier otra ciencia, un paradigma que dio a luz al monopolio de competencia de la disciplina, profesión y ciencia de enfermería. De ahí nace la responsabilidad ética, moral y legal como gestores del cuidado, en el abordaje de los problemas en salud, en este caso particular, de las Lesiones Por Presión. 
Se ha descrito la importancia de seguir construyendo una práctica enfermera holística, multidimensional e integradora que considere a la persona en toda su dimensión ${ }^{(14)}$, puesto que en muchos casos como por ejemplo en el descrito en el presente manuscrito, la etiología de la problemática en salud recae en otras dimensiones: en la esfera social en la que está inmersa la paciente. Lo que a la vista de muchos pareciera un problema físicobiomédico, en la práctica es un problema interdisciplinario, el cual ha sido descrito por la comunidad científica $^{(15)}$, con resultados positivos y como una oportunidad de mejora en el cuidado de las personas mayores.

Otro factor por considerar es la preparación del profesional que proporciona cuidados en los pacientes con LPP, pues se ha constatado $^{(16)}$, que un profesional de enfermería de práctica avanzada presenta una mejora en la optimización de los recursos y en las medidas puestas en marcha para el logro de los objetivos.

Es pertinente abordar precozmente el probable impacto económico de los pacientes con LPP, ya que se ha dejado en manifiesto los grandes costos económicos asociado a dichas lesiones en todos los niveles de atención, lo que configura un importante problema en la salud pública a tener en cuenta por parte de instituciones y sistemas de salud ${ }^{(17)}$, más aún si el paciente se encuentra en su domicilio, por lo que establecer nexos cercanos entre organismos gubernamentales y las familias de dichos pacientes, puede ser un gran buffer para solapar los costes asociados.

\section{CONCLUSIÓN}

El presente manuscrito relevó la importancia de un manejo holístico en las Lesiones Por Presión (LPP), el que puede ser aplicado a otras problemáticas, considerando la dimensión social en la que está inmerso el paciente, un abordaje interdisciplinario, preparación profesional $\mathrm{y}$ abordaje precoz del probable impacto económico de los pacientes con LPP. De esta forma se podrán prevenir futuros inconvenientes en el cumplimiento de los Procesos de Enfermería en el abordaje de problemas de salud asociado a las LPP.

\section{REFERENCIAS BIBLIOGRÁFICAS}

1. NPUAP. National Pressure Ulcer Advisory Panel (NPUAP) announces a change in terminology from pressure ulcer to pressure injury and updates the stages of pressure injury. Washington DC; 2016 [citado 8 octubre 2021]. Disponible en: http://anha.org/uploads/NPUAPStagin g.pdf

2. Asociación Profesional de Enfermeras de Ontario. Valoración y manejo de las lesiones por presión para equipos interprofesionales [Internet]. Tercera edición: RNAO; 2016 [Citado el 08 de octubre 2021]. Disponible en: https://rnao.ca/sites/rnaoca/files/bpg/translations/D0027_Manej o_LPP_2016_final.pdf

3. MINSAL. Chile y Canadá celebran convenio de acuerdo para la implementación de guías de práctica clínica basadas en evidencia [Internet]. minsal.cl. 2017 [citado 8 octubre 2021]. 
Abordaje holístico de las lesiones por presión en la persona mayor: Caso clínico

Disponible

en:

https://www.minsal.cl/minsal-firma-

convenio-de-cooperacion-

internacional-para-implementar-

mejores-practicas-medicas-en-

hospitales-publicos/

4. Valencia-Contrera MA. Invisibilización de enfermería como profesión experta. RchE. 2021 [citado 8 octubre 2021]; 3(1):15-19. Disponible en: https://revistachilenaenfermeria.uchile. cl/index.php/RCHE/article/view/64277

5. World Health Organization. Clasificación estadística internacional de enfermedades y problemas de salud relacionados (CIE) [Internet]. Who.int. 2021 [citado 8 octubre 2021]. Disponible en: https://www.who.int/classifications/cla ssification-of-diseases

6. Ministerio de Salud. Actualización manual de geriatría para médicos. $1^{\mathrm{a}}$ ed. Santiago, Chile: MINSAL; 2019. ISBN: 978-956-348-182-2

7. Carbonell-Fornés $\mathrm{P}$, Murillo-Llorente M. Las úlceras por presión en gerontología: prevalencia y variables definitorias de las lesiones y pacientes. Gerokomos. 2015 [citado 8 octubre 2021];26(2):63-67. Disponible en: https://scielo.isciii.es/pdf/geroko/v26n 2/06_helcos2.pdf

8. Vega Suárez LP; Sánchez Cueto M. Abordaje integral de la enfermera en la prevención de las úlceras por presión. Nuberos Científica. 2018 [citado 8 octubre 2021];3(23):39-46. Disponible en: http://www.indexf.com/nuberos/2017pdf/2339.pdf

9. Henderson V. The nature of nursing: Reflexions after 25 years. New York: N.L.N. Press; 1991.
10. Kouta C, Kaite CP, Papadopoulos I, Phellas CN. Evaluation of Home Care Nursing for Elderly People in Cyprus. 2015;8(2): 376-384. Disponible en: http://internationaljournalofcaringscien ces.org/docs/15_kouta.pdf

11. Herdman H, Kamitsuru S. Diagnósticos enfermeros. Definiciones y clasificación 2021-2023.12a ed. Barcelona: Elsevier España; 2021.

12. Moorhead S, Swanson E, Johnson M, Maas M, Echevarría Pérez P, Morales Moreno I, et al. Clasificación de resultados de enfermería (NOC). $6^{\mathrm{a}}$ ed. Barcelona: Elsevier España; 2018.

13. Butcher H, Bulechek G, Dochterman J, Wagner C. Clasificación de intervenciones en enfermería (NIC). $7^{\mathrm{a}}$ ed. Barcelona: Elsevier España; 2018.

14. Muñoz Begoña M. Calidad de vida de las personas con úlceras por presión. Estudio cualitativo fenomenológico. Index Enferm [Internet]. 2018 Dic [citado 10 de octubre 2021]; 27(4):206210. Disponible en: http://scielo.isciii.es/scielo.php?script= sci_arttext\&pid=S113212962018000300006\&lng=es.

15. Quintana Vargas I, Moreno Miralles A, Tomás Madrid M, Monleón Ruiz M, Montero Bau A, Peris Martí JF. Interdisciplinary approach to the management of medical supplies in the nursing home setting. Farm Hosp. [Internet]. 2017 Ago [citado 10 de octubre de 2021]; 41(4): 497-507. Disponible en: https://dx.doi.org/10.7399/fh.2017.41. 4.10738.

16. Jiménez García JF, Aguilera Manrique G, Arboledas Bellón J, Gutiérrez García M, González Jiménez F, García 
Fernández FP. Efectividad de la enfermera de práctica avanzada en el cuidado de los pacientes con úlceras por presión en atención primaria. Gerokomos [Internet]. 2019 [citado 10 octubre 2021]; 30(1): 28-33. Disponible en: http://scielo.isciii.es/scielo.php?script= sci_arttext\&pid=S1134928X2019000100028\&lng=es.

17. Torra-Bou JE, García-Fernández FP,
Pérez-Acevedo G, Sarabia-Lavin R, Paras-Bravo P, Soldevilla-Ágreda JJ et al. El impacto económico de las lesiones por presión. Revisión bibliográfica integrativa. Gerokomos [Internet]. 2017 [citado 10 de Oct 2021]; 28(2): 83-97. Disponible en:

http://scielo.isciii.es/scielo.php?script= sci_arttext\&pid=S1134928X2017000200083\&lng=es. 